\title{
Symmetry breaking in the formation of magnetic vortex states in a permalloy nanodisk
}

\author{
Mi-Young $\mathrm{Im}^{1}$, Peter Fischer ${ }^{1}$, Keisuke Yamada ${ }^{2}$, Tomonori Sato ${ }^{3}$, Shinya Kasai ${ }^{4}$, \\ Yoshinobu Nakatani ${ }^{3} \&$ Teruo Ono ${ }^{2}$
}

The magnetic vortex in nanopatterned elements is currently attracting enormous interest. A priori, one would assume that the formation of magnetic vortex states should exhibit a perfect symmetry, because the magnetic vortex has four degenerate states. Here we show the first direct observation of an asymmetric phenomenon in the formation process of vortex states in a permalloy nanodisk using high-resolution full-field magnetic transmission soft X-ray microscopy. Micromagnetic simulations confirm that the intrinsic Dzyaloshinskii-Moriya interaction, which arises from the spin-orbit coupling due to the lack of inversion symmetry near the disk surface, as well as surface-related extrinsic factors, is decisive for the asymmetric formation of vortex states.

\footnotetext{
${ }^{1}$ Center for X-ray Optics, Lawrence Berkeley National Laboratory, Berkeley, California 94720, USA. ${ }^{2}$ Institute for Chemical Research, Kyoto University, Uji 611-0011, Japan. ${ }^{3}$ Graduate School of Informatics and Engineering, University of Electro-Communications, Chofu 182-8585, Japan. ${ }^{4}$ Spintronics Group, Magnetic Material Center, National Institute for Materials Science (NIMS), Tukuba 305-0047, Japan. Correspondence and request for materials should be addressed to M.-Y.I (email: mim@lbl.gov).
} 
$\mathrm{V}$ ortices and antivortices are topological excitations with a half-integer skyrmion charge of $q=|n p / 2|=1 / 2$, where $n$ is the winding number and $p$ is the polarity. Vortices have a winding number of $n=+1$ and a polarity of $p= \pm 1$, whereas antivortices have $n=-1$ (refs 1-3). Magnetic vortices are characterized by an in-plane curling magnetization (chirality) and a nanometer-sized central region with an out-of-plane magnetization (polarity) ${ }^{4-6}$. The latter is defined by a clockwise $(c=1)$ or counter-clockwise $(c=-1)$ rotation of the in-plane magnetization. The polarity is the orientation of the core magnetization, either pointing up $(p=1)$ or down $(p=-1)$. With two possible orientations of $c$ and $p$, four different ground states of a magnetic vortex can occur ${ }^{7,8}$. The magnetic vortex structure constitutes a fascinating topological structure for fundamental studies of nanoscale spin behaviour and offers great potential as a novel concept in data storage technologies ${ }^{9-15}$. A priori, one would expect the energy of the four states to be degenerate. A non-degeneracy would not only constitute an unconventional physical phenomenon at the nanoscale but could also lead to potentially interesting applications of magnetic vortices with regard to magnetic sensor or logic elements ${ }^{16,17}$. Owing to the lack of experimental techniques enabling simultaneous imaging of in-plane $(c)$ and out-of-plane $(p)$ magnetic components in nanopatterned structures, most of the experiments on magnetic vortices reported so far have focussed on either the chirality or polarity ${ }^{9,15}$. Therefore, the degeneracy of a magnetic vortex has not been experimentally verified to date.

Here we report the first experimental result for symmetry breaking in the formation process of vortex states (VSs) in a single circular permalloy $\left(\mathrm{Py}, \mathrm{Ni}_{80} \mathrm{Fe}_{20}\right)$ nanodisk. The origin of the experimentally observed asymmetric phenomenon is interpreted as the combination of an 'intrinsic' Dzyaloshinskii-Moriya interaction (DMI) arising from the spin-orbit coupling ${ }^{17,18}$ and surface-related 'extrinsic' factors, such as edge defects, surface roughness, and so on. We utilize full-field magnetic transmission soft X-ray microscopy (MTXM) with high-spatial resolution down to $20 \mathrm{~nm}$ (ref. 19), because the use of X-ray magnetic circular dichroism for magnetic contrast allow us to observe chirality and polarity simultaneously in a large array of permalloy $\left(\mathrm{Py}, \mathrm{Ni}_{80} \mathrm{Fe}_{20}\right)$ disks and, therefore, to address the formation process of magnetic VSs in applied external magnetic fields.

\section{Results}

Imaging of magnetic vortex structures. Figure 1 shows MTXM images of the in-plane (Fig. 1a) and out-of-plane (Fig. 1b) magnetic configurations in an identical area of $100-\mathrm{nm}$ thick Py nanodisks with a radius $r=500 \mathrm{~nm}$. The dark/bright contrast indicates the projection of the local Fe magnetization along the photon propagation direction of circularly polarized photons. Consequently, the curling in-plane domain structure (chirality), which rotates either clockwise or counter-clockwise, and the small dark or bright spot in the centre of the nanodisks, which represents the upward or downward pointing vortex core (polarity), are clearly visible in Fig. 1a,b. The images were taken at the remanent state $(0 \mathrm{mT})$ after sweeping the external magnetic field from 100 to $0 \mathrm{mT}$ in field steps of $5 \mathrm{mT}$. The complete vortex configuration of each nanodisk is illustrated in Fig. 1c, where the sequence of the colours indicates the rotation direction of the magnetization in the plane of the nanodisks, and the black and white spots in the centre represent the direction of the vortex core. Obviously, four different VSs, which are defined as $c=1: p=1(\mathrm{~S} 1), c=-1: p=-1(\mathrm{~S} 2), c=-1$ : $p=1(\mathrm{~S} 3)$, and $c=1: p=-1(\mathrm{~S} 4)$, are generated in the nanodisks. As the nanodisks are in close proximity to each other with a centreto-centre distance of $d=1,150 \mathrm{~nm}$, one can expect that the dipolar interactions with nearby nanodisks affect the VS formation of each nanodisk within an array. To clarify that assumption, we have studied experimentally the magnetization process of nanodisk arrays with
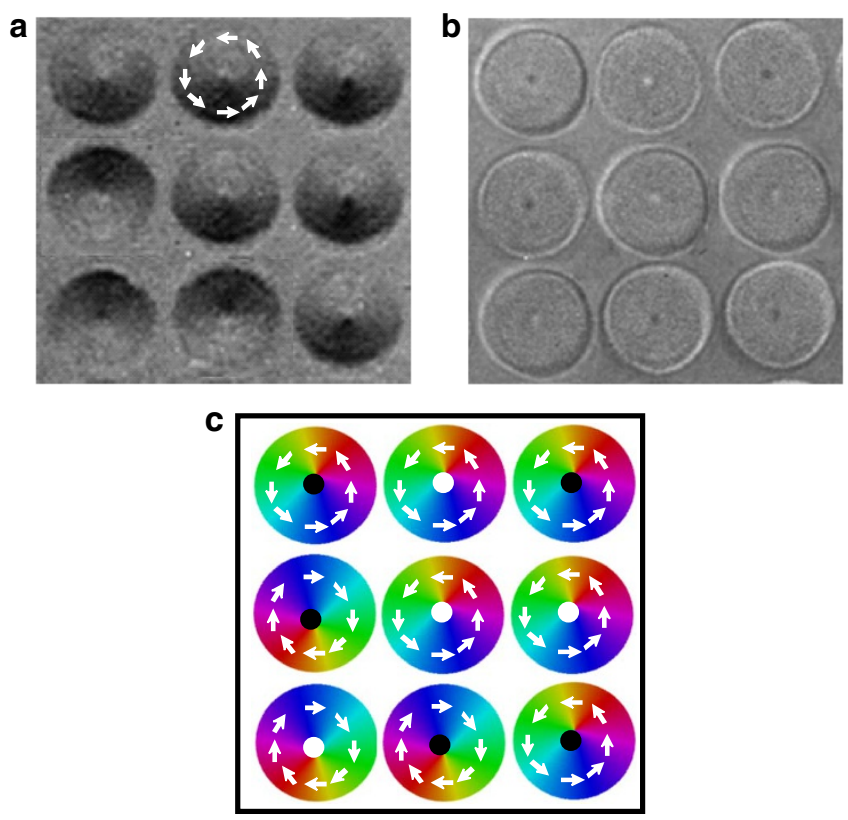

Figure 1 | Experimental images of vortex structures in Py nanodisk array. MTXM images of in-plane (a) and out-of-plane (b) magnetic components taken at the remanent state in a $100-n m$-thick nanodisks. All images were observed in the identical array area. The sense of rotation of the in-plane magnetization is indicated by a white arrow (a). The black and white spots of the central regions in nanodisks are upward and downward vortex cores, respectively (b). The complete vortex configuration of each nanodisk is also illustrated (c).

different centre-to-centre distances of $d=1200,1500,1800 \mathrm{~nm}$. We observed that, with decreasing centre-to-centre distance, the external field for the nucleation of vortices also slightly decreases and the magnetization of nanodisks seems to proceed collectively. One might argue that this results from the dipolar coupling of the nanodisks, but, interestingly, we do not observe the expected correlation in vortex structures of neighbouring nanodisks with regard to the rotation of $c$ and the orientation of $p$ as a function of the centre-to-centre distance. We conclude that dipolar interactions do not have a strong impact on determining the types of VS in individual nanodisks within an array.

The complete magnetization-reversal process of Py nanodisks was observed with varying magnetic fields, and a sequence of typical domain structures at magnetic fields from 100, 40, 20, 0, - 20, -40, and $-100 \mathrm{mT}$ are shown in Fig. 2a. At an external magnetic field of $100 \mathrm{mT}$ pointing in the positive $x$ direction (indicated by the arrow), the nanodisks are fully saturated. By sweeping the applied magnetic field from the saturation field back to $0 \mathrm{mT}$, magnetic vortices are nucleated, with the vortex cores moving from the edge of the disk towards the centre at $0 \mathrm{mT}$. With the external field progressing from 0 to $-100 \mathrm{mT}$, the vortex cores shift from the centre to the opposite edges, where they finally annihilate.

Symmetry breaking in the formation of vortex states. To investigate the symmetric character of the formation process of VSs in individual disks with high statistical significance, we repeatedly recorded MTXM images throughout the process of magnetization reversal in the nanodisk arrays. Figure $2 \mathrm{~b}$ displays a representative ensemble of eight repeated experiments for $c$ and $p$ in two neighbouring nanodisks, corresponding to 16 independent measurements. Most interestingly, we found that certain types of VSs are dominantly generated, in particular, the formation probability of VS 
a

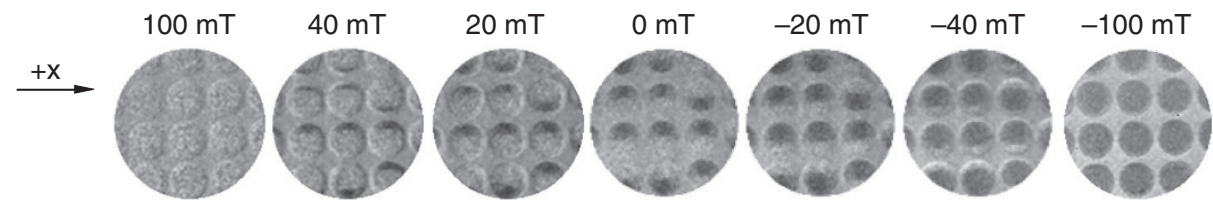

b

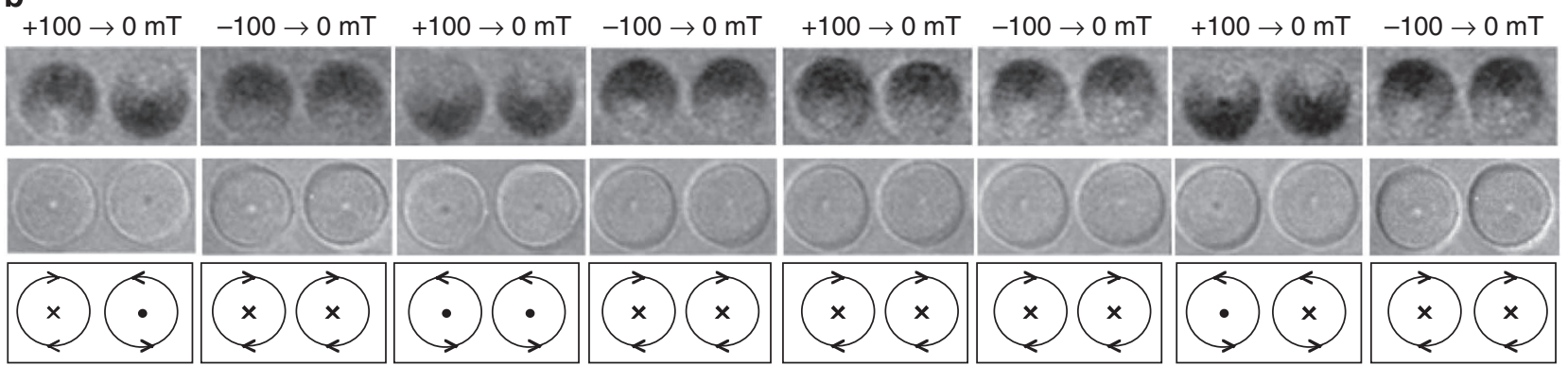

Figure 2 | Formation process of VSs in repeated measurements. (a) Image sequence of vortex-creation and -annihilation processes driven by an external magnetic field. (b) Chirality and polarity images taken from eight representative images showing two individual nanodisks with a radius of $500 \mathrm{~nm}$ and a thickness of $100 \mathrm{~nm}$. The generation rate of each VS group, which is defined as the number of times that each VS group is generated relative to the total number of repetitions, is obviously asymmetric. VSs with a signature of $c p=-1$ are predominantly generated compared to VSs with $c p=1$ with a quantitative generation ratio of 8:0 and 7:1 in the first and second nanodisk, respectively.

Table 1 | The averaged experimental generation probabilities of vortex state (VS).

\begin{tabular}{|c|c|c|c|c|c|c|}
\hline Generation Prob. & $0.71 \pm 0.08$ & $0.30 \pm 0.06$ & $0.51 \pm 0.08$ & $0.48 \pm 0.09$ & $0.47 \pm 0.10$ & $0.52 \pm 0.08$ \\
\hline
\end{tabular}

groups with $c p=1$ (S1, S2: left-handed) and $c p=-1$ (S3, S4: righthanded) is asymmetric in each nanodisk.

To enhance the statistical significance, a total of 50 repeated observations in an array of 30 nanodisks, which yields a total of 1,500 independent measurements, was analysed. Although we found that the dominantly generated VS group for an individual nanodisk can be either $c p=1$ or $c p=-1$, we always found an asymmetric generation ratio for the two VS groups. In Table 1, we list the averaged generation probabilities of VSs, where dominantly (recessively) formed VS groups are denoted as majority (minority). The generation probabilities of majority and minority are found to be $0.71 \pm 0.08$ and $0.30 \pm 0.06$, obviously indicating an asymmetry. On the other hand, as also shown in Table 1, the two VSs belonging to the same VS $c p$-group are almost symmetrically generated. This result clearly confirms that an experimentally observed symmetrybreaking phenomenon occurs in the formation of two VS groups with different handednesses. This is completely unexpected, assuming that there is equal probability, regardless of handedness, in a single circular nanodisk to generate the four different VSs.

3D micromagnetic modelling. To interpret and explain the asymmetric process of VS formation, we took the DMI into consideration, because it inevitably arises from the spin-orbital coupling due to the broken inversion symmetry at surfaces/interfaces in magnetic thin layers ${ }^{17,18}$. The DMI has been discussed as the origin for spin spiral textures and has been theoretically predicted to affect the shape and size of magnetic vortices ${ }^{20,21}$. To explore the effect of DMI on the asymmetric formation process of VS, we calculated the generation probabilities of each VS by performing a micromagnetic simulation of the magnetization process of a nanodisk from saturated state to zero-field state. The simulation was carried out with our original code, which was expanded to include the DMI term in the following form:

$$
\begin{aligned}
E= & A_{\mathrm{ex}} \frac{1}{M_{\mathrm{s}}^{2}}\left[\left(\frac{\partial M_{\mathrm{x}}}{\partial x}\right)^{2}+\left(\frac{\partial M_{\mathrm{y}}}{\partial y}\right)^{2}+\left(\frac{\partial M_{\mathrm{z}}}{\partial z}\right)^{2}\right] \\
& -\frac{1}{2} \mu_{0} \mathbf{M} \cdot \mathbf{H}_{\mathrm{ms}}+e_{D} .
\end{aligned}
$$

The first and second terms correspond, respectively, to the exchange energy and the magnetostatic energy with the stray field $\mathbf{H}_{\mathrm{ms}}$. The last term is DM energy given as

$$
e_{D}=D \frac{1}{M_{\mathrm{s}}^{2}}\left[M_{\mathrm{z}}\left(\frac{\partial M_{\mathrm{x}}}{\partial y}-\frac{\partial M_{\mathrm{y}}}{\partial x}\right)-M_{\mathrm{x}} \frac{\partial M_{\mathrm{z}}}{\partial y}+M_{\mathrm{y}} \frac{\partial M_{\mathrm{z}}}{\partial x}\right] \text {, }
$$

where $D$ is the Dzyaloshinskii constant, which determines the magnitude of the DMI and its sign specifies the orientation of spin spiral state owing to the DMI 18,20 . For this simulation, we adapted a three-dimensional (3D) model where the DMI exists only on the disk surfaces (top, bottom and lateral) because the DMI is considered to be localized at the surface ${ }^{18,22-24}$. A schematic diagram of the $3 \mathrm{D}$ modelling is shown in Fig. 3. The model system is a Py circular disk with $r=500 \mathrm{~nm}$ and $h=100 \mathrm{~nm}$ (Fig. 3a), which resembles the physical dimensions of the actual disks used in the experiment of Figs 1 and 2. The mesh size is $4 \times 4 \times 6.25 \mathrm{~nm}^{3}$ and the DMI is assumed to exist only in the outer meshes, which are shaded in yellow in the side (b) and top view (c) diagrams of Fig. 3. The coordinates of the DMI components on the lateral and top/bottom surfaces are indicated. In our 3D model, the DMI term on the lateral surface is given in the radial direction, whereas the DMI term on 


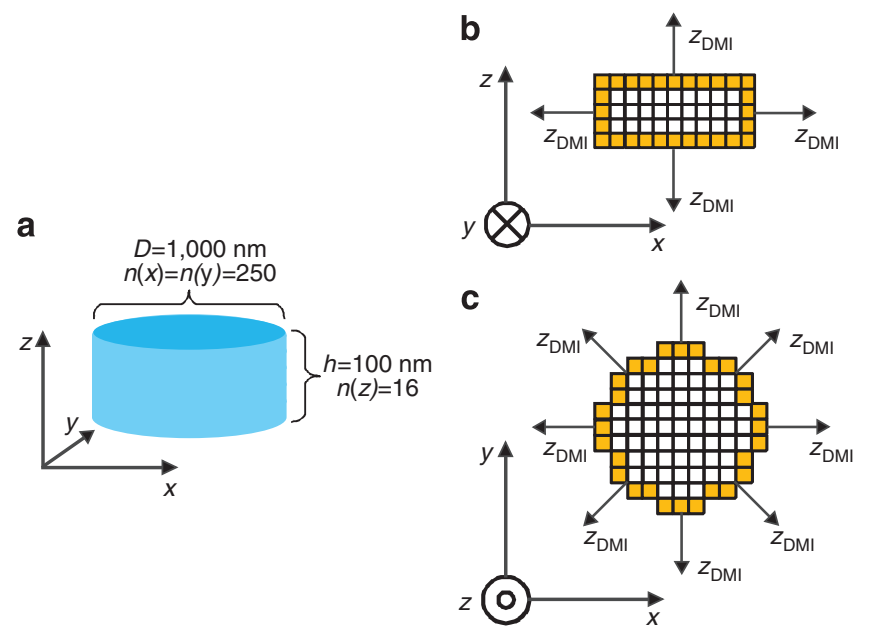

Figure $\mathbf{3}$ | Schematic diagram of 3D modelling. (a) The model system is a Permalloy nanodisk with the radius of $500 \mathrm{~nm}$ and the thickness of $100 \mathrm{~nm}$ divided into a mesh with an element size of $4 \times 4 \times 6.25 \mathrm{~nm}^{3}$. In this model, the DMI is considered to exist only in the outer elements shaded in yellow as shown in side (b) and top (c) view diagrams. The coordinates of the DMI terms on the lateral and top/bottom surfaces given in our 3D model are indicated.

the top/bottom surfaces is given in the $z$ - and angular coordinates in the local cylindrical system. Each coordinate of the DMI on the lateral surface and the DMI on the top/bottom surfaces corresponds to the out-of-plane and in-plane coordinate, respectively. As material parameters for Py, we used $M_{\mathrm{s}}=8.6 \times 10^{5} \mathrm{~A} \mathrm{~m}^{-1}$, exchange stiffness $A_{\mathrm{ex}}=1.3 \times 10^{-11} \mathrm{Jm}^{-1}$, zero magnetocrystalline anisotropy, and damping constant $\alpha=1.00$.

Simulation for the generation probability of each VS. To investigate the generation probability of each VS under the influence of the DMI, we chose the initial magnetization structure to be a singledomain structure pointing into the same direction (saturated state) by an applied external magnetic field of $150 \mathrm{mT}$. The external field was decreased from $150 \mathrm{mT}$ with a sweep rate of $1 \mathrm{mT} \mathrm{ns}^{-1}$ until the final state was completed. In the experiment, the change of the magnetization structure was gradual because of the very slow magnetic field sweep rate. On the other hand, a very fast sweep rate had to be used in the simulation because of the limitation of the calculation time. We used a Gilbert damping constant $\alpha$ of 1 to make the change of the magnetization structure gradual with a fast sweep rate, thereby mimicking the experiment. Figure 4 illustrates representative image sequences of magnetic structures for the final VS of $c=-1$ : $p=-1$ (S2) simulated at $D=1.03 \times 10^{-3} \mathrm{~J} \mathrm{~m}^{-2}$. The in-plane (left) and out-of-plane (right) magnetization structures observed on the top (a) and bottom (b) layers at each step are displayed, respectively. Interestingly, this simulation provides great detail about the magnetization process of nanodisks, which so far has been completely unexplored. The nucleation of magnetic vortices on the top and bottom surfaces occur asynchronous and unevenly. The cores nucleate from the left side of the bottom layer first $(t=87.6 \mathrm{~ns})$, followed by multiple cores appearing on both layers $(t=103.8 \mathrm{~ns})$. As the magnetization proceeds, a single core with opposite polarity remains on the top and bottom layers ( $t=108 \mathrm{~ns})$ and the magnetic structures on both layers become identical. Finally, one of the cores of either the top layer or the bottom layer switches $(t=142.2 \mathrm{~ns})$ and the final VS is reached. By varying the DM constant in the simulation, including the case of $D=0$, we have systematically investigated the nucleation mechanism and magnetization process of VS. At $D=0$, the generation position of cores are quite random such as left side, right-upper,

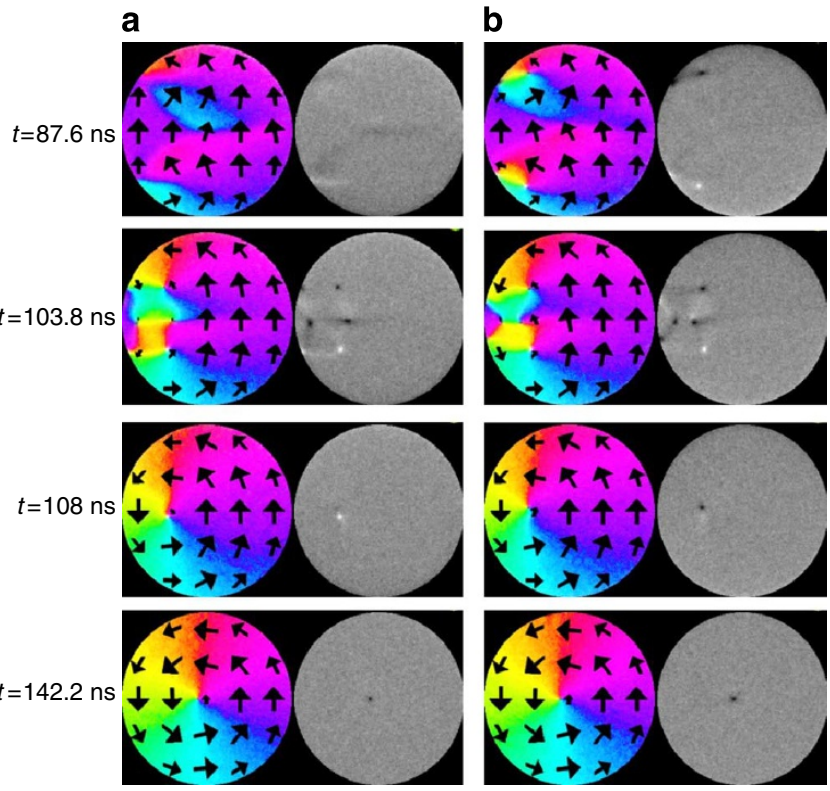

Figure 4 | Simulated magnetic structures during the magnetization process of a nanodisk. Typical image series of magnetic structures obtained by the simulation for the magnetization process of a nanodisk. In-plane (left) and out-of-plane (right) magnetic structures at each field (time) step are respectively displayed, which are simulated on the top (a) and the bottom layer (b). In the out-of-plane images, the black and white spots represent the down and up polarizations, respectively.

left-down of disk, and so on. The overall magnetization behaviour of VS sensitively depends on the initial nucleation stage of cores. When the cores appear from one side of disk, the magnetization of VS mostly accompanies with appearing of multiple cores in random positions of disk, while the magnetization proceeds with the moving of created cores when the cores generate on both sides of disk. In the absence of DMI, the same final VS can be defined by different nucleation and magnetization behaviours. At $D \neq 0$, the nucleation mechanism is rather simple (Supplementary Movies 1), which might be due to the DMI causing selectivity. The details of the nucleation stage, configuration of multiple cores, motions of cores, annihilating process, and the mechanism of polarity determination can be different, depending on the DM constant. To examine the generation probabilities of each VS, the simulation for the nanodisk magnetization process was repeatedly carried out. We also included thermal noise at $\mathrm{T}=300 \mathrm{~K}$ (ref. 25) to introduce stochasticity into our model. The simulation was repeated 200 times for each DM constant and the generation probabilities of $c p=1$ or -1 were calculated to match the experimental approach. In addition to the simulation with the DMI included on all surfaces, we have also performed the simulation for the case of the DMI existing only on the lateral surface or only on the top and bottom surfaces, respectively. We found that the nucleation mechanism, formation process, and the generation probability of VS are considerably different in each case (Supplementary Fig. S1 and Supplementary Table S1). The results obtained by the simulation with the DMI on all surfaces show comprehensive nucleation and formation processes driven by contributions of all DMI on the lateral surface and top/bottom surfaces.

\section{Discussion}

The simulation results are summarized in Table 2 . The generation probabilities of the VS groups of $c p=1$ and $c p=-1$ are asymmetric and the value of the asymmetry scales with the DM constant. This clearly demonstrates that the DMI has a strong impact on the 


\section{Table 2 | Simulated generation probabilities of vortex states (VS).}

\begin{tabular}{lcc} 
DM const. $\left(\times \mathbf{1 0} \mathbf{- 3}^{-\mathbf{3}} \mathbf{~ \mathbf { m } ^ { - 2 }}\right)$ & \multicolumn{2}{c}{ Generation Prob. } \\
\cline { 2 - 3 } & $\mathbf{c p = 1}$ & $\mathbf{c p = - 1}$ \\
\hline 0.9 & 0.45 & 0.55 \\
1.03 & 0.36 & 0.64 \\
1.22 & 0.3 & 0.7 \\
1.84 & 0 & 1
\end{tabular}

The generation probabilities of VS with different handedness as function of variable DM constant taken from a total of 800 3D simulations.

asymmetric generation of $c p=1$ or -1 . Remarkably, we observe in the simulation that the in-plane magnetization always proceeds in such a way that the chirality with counter-clockwise rotation sense $(c=-1)$ is generated irrespective of the size of the DM constant and the final state, thereby establishing an unprecedented phenomenon that is due to the DMI. This simulation finding is inconsistent with the experimental result, which observes both chiralities of $c=-1$ and $c=1$ (Figs 1 and 2). This difference strongly suggests that surface-related 'external' factors such as edge defects, roughness and geometric variations of individual disks, and so on, might also have an impact on the experimentally observed results in real nanodisks ${ }^{25}$. Thus, the experimental observation that the preferred VS group is different in some nanodisks can be attributed to surface-related external factors to the formation process of VS. Such surface-related defects, which happen to occur inevitably and randomly in individual nanodisks, will affect the magnetization process in particular the nucleation mechanism, which is crucial for the determination of the final state. Therefore, we conclude that both the 'intrinsic' DMI and the surface-related 'extrinsic' factors are important aspects to explain the experimentally observed asymmetric generation behaviour of VS.

To summarize, we have experimentally shown that VSs in a single magnetic nanodisk experience a symmetry breaking in the formation process, which is contrary to the a priori assumption that a magnetic vortex has four different but degenerate ground states. This asymmetric formation process of VS is essential to precisely control the magnetic/spin process in magnetic vortices, which is needed to realize vortex-based data-storage devices. We interpret our experimentally observed asymmetric phenomenon as a consequence of the 'intrinsic' DMI, but we also take surface-related 'extrinsic' factors such as edge defects and/or roughness, ans so on into account. Our results might impact both the fundamental understanding of nanoscale spin behaviour and its potential for technological applications.

\footnotetext{
Methods

Sample preparation and MTXM measurement. Permalloy (Py) nanodisks with a radius of $500 \mathrm{~nm}$ and a thickness of $100 \mathrm{~nm}$ were patterned by electron-beam lithography and lift-off process. A total of $30 \times 30$ nanodisk arrays with a centreto-centre distance of $d=1,150,1,200,1,500$ and $1,800 \mathrm{~nm}$ were deposited on 100-nm-thick silicon-nitride membranes to allow for sufficient transmission of soft X-rays. Permalloy films were prepared by means of evaporation in an ultrahigh vacuum using an electron-beam gun at a pressure lower than $1 \times 10^{-7}$ Torr. The disks exhibit a polycrystalline structure. Direct observation of vortex structures in Py nanodisks was carried out using full-field MTXM at the Advanced Light Source (beamline 6.1.2.) ${ }^{19}$, enabling real-space magnetic images with high-spatial resolution down to $20 \mathrm{~nm}$. Within an exposure time of a few seconds, a full array of $30 \times 30$ disks can be imaged. Magnetic imaging of Py nanodisks was performed at a photon energy corresponding to the $\mathrm{Fe}_{3}(707 \mathrm{eV}) \mathrm{X}$-ray absorption edge. In MTXM, magnetic contrast is given by X-ray magnetic circular dichroism arising from the dependence of the X-ray absorption coefficients on the orientation between magnetization and photon helicity. To record images of in-plane and out-of-plane magnetizations, specifically the chirality and polarity in the Py nanodisk, the array was mounted at 60 and $90^{\circ}$ angles with respect to the X-ray propagation direction, respectively. To reduce non-magnetic
}

background for the in-plane images, images taken at certain fields were normalized to an image recorded in the fully saturated state. The magnetic contrast of the vortex core (out-of-plane configuration) was enhanced by taking images with opposite photon helicities and dividing the two, providing a clear core image with smooth background.

\section{References}

1. Tretiakov, O. A. \& Tchernyshyov, O. Vortices in thin ferromagnetic films and the skyrmion number. Phys. Rev. B 75, 012408 (2007).

2. Ezawa, M. Compact merons and skyrmions in thin chiral magnetic films. Phys. Rev. B 83, 100408 (2011).

3. Hertel, R. \& Schneider, C. M. Exchange explosions: magnetization dynamics during vortex-antivortex annihilation. Phys. Rev. Lett. 97, 177202 (2006).

4. Shinjo, T., Okuno, T., Hassdorf, R., Shigeto, K. \& Ono, T. Magnetic vortex core observation in circular dots of Permalloy. Science 289, 930-932 (2000).

5. Wachowiak, A. et al. Direct observation of internal spin structure of magnetic vortex cores. Science 298, 577-579 (2002).

6. Fischer, P. et al. X-ray imaging of magnetic vortices in confined magnetic structures. Phys. Rev. B 83, 212402 (2011).

7. Guslienko, K. Y. et al. Field evolution of magnetic vortex state in ferromagnetic disks. Appl. Phys. Lett. 78, 3848-3850 (2001).

8. Guslienko, K. Y. et al. Eigenfrequencies of vortex state excitations in magnetic sumbmicron-size disks. J. Appl. Phys. 91, 8037-8039 (2002).

9. Choe, S.- B. et al. Vortex core-driven magnetization dynamics. Science 304, 420-422 (2004)

10. S. Novosad, V. et al. Magnetic vortex resonance in patterned ferromagnetic dots. Phys. Rev. B 72, 024455 (2005).

11. Van Waeyenberge, B. et al. Magnetic vortex core reversal by excitation with short bursts of an alternating field. Nature 444, 461-464 (2006).

12. Yamada, K. et al. Electrical switching of the vortex core in a magnetic disk. Nature Mater. 6, 269-273 (2007).

13. Kasai, S. et al. Probing the spin polarization of current by soft X-ray imaging of current-induced magnetic vortex dynamics. Phys. Rev. Lett. 101, 237203 (2008).

14. Lee, K.- S. et al. Universal criterion and phase diagram for switching a magnetic vortex core in soft magnetic nanodots. Phys. Rev. Lett. 101, 267206 (2008).

15. Vansteenkiste, A. et al. X-ray imaging of the dymanic magnetic vortex core deformation. Nature Phys. 5, 332-334 (2009).

16. Curcic, M. et al. Polarization selective vortex dynamics and core reversal in rotating magnetic fields. Phys. Rev. Lett. 101, 197204 (2008).

17. Bogdanov, A. N. \& Rößler, U. K. Chiral symmetry breaking in magnetic thin films and multilayers. Phys. Rev. Lett. 87, 037203 (2001).

18. Bode, M. et al. Chiral magnetic order at surfaces driven by inversion asymmetry. Nature 447, 190-193 (2007).

19. Fischer, P. Soft X-ray microscopy-a powerful analytical tool to image magnetism down to fundamental length and time scales. AAPPS bulletin 18, 12-17 (2008).

20. Butenko, A. B., Leonov, A. A., Bogdanov, A. N. \& Rößler, U. K. Theory of vortex state in magnetic nanodisks with induced Dzyaloshinskii-Moriya interaction. Phys. Rev. B 80, 134410 (2009).

21. Butenko, A. B., Leonov, A. A., Bogdanov, A. N. \& Rößler, U. K. Influence of the Dzyaloshinskii-Moriya interaction on vortex states in magnetic nanodisks. J. Phys.: Conf. Ser. 200, 042012 (2010).

22. Heinze, S. et al. Spontaneous atomic-scale magnetic skyrmion lattice in two dimensions. Nature Phys. 7, 713-718 (2011).

23. Ferriani, P. et al. Atomic-scale spin spiral with a unique rotational sense: Mn monolayer on W(001). Phys. Rev. Lett. 101, 027201 (2008).

24. Meckler, S. et al. Real-space observation of right-rotating inhomogeneous cycloidal spin spiral by spin-polarized scanning tunneling microscopy in a triple axes vector magnet. Phys. Rev. Lett. 103, 157201 (2009).

25. Nakatani, Y., Uesaka, Y., Hayashi, N. \& Fukushima, H. Computer simulation of thermal fluctuation of fine particle magnetization based on Langevin equation. J. Magn. Magn. Mater. 168, 347-351 (1997).

26. Vansteenkiste, A. et al. Chiral symmetry breaking of magnetic vortices by sample roughness. New J. Phys. 11, 063006 (2009).

\section{Acknowledgements}

This work was supported by the Director, Office of Science, Office of Basic Energy Sciences, of the U.S. Department of Energy under Contract No. DE-AC02-05CH11231 and partly supported by a Grant-in-Aid for Scientific Research (S) from the Japan Society for the Promotion of Science and the Collaborative Research Program of Institute for Chemical Research, Kyoto University.

\section{Author contributions}

M.Y.I. designed the research and performed experiments. M.-Y.I., P.F. and T.O. analysed and interpreted the results. K.Y and S.K. prepared samples. Y.N. and T.S. developed the 
model and carried out simulations. M.-Y.I and P.F. wrote the manuscript. All authors commended on the final manuscript.

\section{Additional information}

Supplementary Information accompanies this paper at http://www.nature.com/ naturecommunications
Competing financial interests: The authors declare no competing financial interests.

Reprints and permission information is available online at http://npg.nature.com/ reprintsandpermissions/

How to cite this article: Im, M-Y. et al. Symmetry breaking in the formation of magnetic vortex states in a permalloy nanodisk. Nat. Commun. 3:983 doi: 10.1038/ncomms1978 (2012). 